\title{
Variability in Clinical Practice Guidelines for Sweetening Agents in Newborn Infants Undergoing Painful Procedures
}

\author{
Anna Taddio, PhD, ${ }^{\dagger}$ Ashley Yiu, $\dagger$ Ryan W. Smith, MSc, $\dagger$ Joel Katz, PhD, + \\ Carol McNair, CNS-NNP,§ and Vibhuti Shah, MD\|
}

\begin{abstract}
Objective: Sweetening agents have been recommended in position statements and consensus documents for procedural pain management in neonates; however, it is not clear if this has resulted in widespread adoption in clinical practice. The objective of this study was to investigate unit-specific protocols for the use of sweetening agents.
\end{abstract}

Methods: Structured telephone survey with qualified personnel in special care (level II) nurseries and neonatal intensive care (level III) units across Canada. The frequency and pattern of recommended use of sweetening agents was documented.

Results: Eighty-six of 92 units $(93.5 \%)$ participated. Sixty-four percent recommended sucrose and $2.3 \%$ recommended glucose for procedural pain management; $87.7 \%$ had a guideline. Sweetening agents were most commonly recommended for venipuncture/ venous cannulation $(91.2 \%$ for both), lumbar puncture $(87.7 \%)$, and heel lance $(82.5 \%)$. Dosing guidelines ranged from $0.05 \mathrm{~mL}$ of $24 \%$ sucrose solution to $3 \mathrm{~mL}$ of $25 \%$ sucrose solution. Sweeteners were not recommended for infants with necrotizing enterocolitis $(77.2 \%)$ or those who were nil per os $(75 \%)$.

Conclusions: Sweetening agents were recommended for procedural pain management in two-thirds of special care nurseries and neonatal intensive care units across Canada with extensive variability in specific dosing guidelines. Audits of pain management practices should therefore account for unit-specific practice guidelines.

Key Words: sucrose, practice guidelines, pain management, infant/ newborn
Received for publication May 7, 2008; revised June 21, 2008; accepted June 23, 2008.

From the *Department of Pharmacy Practice; $\dagger$ Leslie Dan Faculty of Pharmacy, University of Toronto; \$Department of Psychology, York University; §Department of Paediatrics, The Hospital for Sick Children; and $\|$ Department of Paediatrics, Mount Sinai Hospital, Toronto, Ontario.

Funding: This study was supported by a Canadian Institutes of Health Research (CIHR) research studentship to Ms A Yiu, a CIHR New Investigator Award to Dr A Taddio, and a CIHR Canada Research Chair in Health Psychology to Dr J Katz. The funding did not include any input into the design and conduct of the study; collection, management, analysis, or interpretation of data; preparation, review, or approval of manuscript. There are no conflicts of interest.

Reprints: Anna Taddio, PhD, Department of Pharmacy Practice, Leslie Dan Faculty of Pharmacy, University of Toronto, 144 College Street, Toronto, Ontario, M5S 3M2, Canada (e-mail: anna.taddio@, utoronto.ca).
$\mathrm{N}$ ewborn infants in special care (level II) nurseries and intensive care (level III) units are exposed to many painful medical procedures as part of clinical care. ${ }^{1}$ Not only do these procedures cause acute pain, but when performed repeatedly, lead to long-term changes in the central nervous system that causes alterations in the processing of subsequent painful stimuli. ${ }^{2,3}$ As a result, pain prevention and management strategies have been identified as a priority for this patient population. ${ }^{4}$

Dozens of clinical trials have demonstrated analgesic effects from sweet-tasting substances such as sucrose when administered to newborn infants during painful medical procedures. ${ }^{5}$ Although not fully understood, the underlying mechanism of action is hypothesized to involve activation of the endogenous opioid system by sweet taste. ${ }^{6}$ Sucrose has been the most widely studied of the sweetening agents currently in use for the relief of pain, followed by glucose. Between 2000 and 2006, several consensus documents and position statements by pain researchers and national organizations [American Academy of Pediatrics (AAP) and Canadian Paediatric Society (CPS)] were published recommending routine use of sucrose for the management of procedural pain in neonates. ${ }^{4,7-10}$

It is not known if these recommendations have been adopted by special care nurseries and neonatal intensive care units. In the only previous national survey of neonatal unit protocols for analgesia conducted in Australia in $2004,{ }^{11} 23 \%$ of units reported using sucrose or other sweettasting solutions. That study was conducted before publication of a large systematic review and AAP and CPS position statements. ${ }^{5,9}$ The objective of the present study was to determine current practice guidelines for the use of sweetening agents in newborn infants undergoing painful medical procedures in Canadian special care nurseries and neonatal intensive care units.

\section{MATERIALS AND METHODS}

We conducted a Canada-wide survey of special care nurseries and neonatal intensive care units regarding their clinical practice guidelines for sweetening agents. Potential units were identified from the provincial ministries of health for each province. Using a structured telephone interview, a trained interviewer questioned the charge nurse, nurse educator, or qualified designate in each unit about the use of sweet-tasting solutions and unit characteristics (such as number of inpatient beds, type of newborn care services provided, number of attending physicians). Potential respondents were identified by senior investigators (via local and national research and clinical networks) or by the units themselves (during first telephone contact by the 
interviewer). All respondents verified their qualification to answer questions before their participation. The survey was designed according to standard survey construction guidelines and included mainly closed-ended questions. ${ }^{12}$ It was pretested for clarity and completeness on respondents from 3 local institutions. The time needed to administer the survey was approximately 10 minutes. For units that used sweeteners, respondents mailed or faxed their protocols to the investigators to verify responses. The study was approved by the ethics boards of the University of Toronto and The Hospital for Sick Children. The study was explained and verbal consent was obtained from respondents before participation.

\section{SAMPLE SIZE AND STATISTICAL ANALYSIS}

We estimated a sample size of 100 units, with $23 \%$ having unit-specific protocols for sweeteners (as per the prior survey). ${ }^{11}$ This resulted in a precision of approximately $10 \% .^{13}$ Logistic regression was performed to identify factors predictive of sweetening agent utilization, including: type of unit; number of inpatient beds; number of physicians; presence of a pain committee; presence of nurse practitioners; and a pain champion. Categorical data were reported as frequency (percent) and continuous data were reported as mean (SD) or mode (range). Data were analyzed using the statistical software package SPSS (v.15.0, Chicago, IL). A $P$ value of $\leq 0.05$ was considered significant.

\section{RESULTS}

The study was conducted between June 12, 2007 and September 19, 2007. Out of 98 potential units identified by the provincial ministries of health, 92 qualified; in excluded units, neonatal special care and intensive care services were not being provided. In 4 cases, respondents could not be reached after multiple attempts, and in 2 cases, respondents declined participation. Therefore, interviews were conducted with respondents from $86(93.5 \%)$ units.

Respondents included 33 unit managers, 19 nurse educators, 13 charge nurses, 10 pain committee members, 6 nurse practitioners, and 1 neonatologist. Fifty-two $(60.5 \%)$ units contained special care nurseries, $3(3.5 \%)$ contained neonatal intensive care units, and the remaining 31 (36\%) contained both. The mean (SD) number of inpatient beds was 20 (14).

Overall, $55(64 \%)$ of units had a practice guideline in place for the use of sucrose and $2(2.3 \%)$ for another sweetener (glucose). Fifty $(87.7 \%)$ units with a written protocol provided it to investigators. Most of the time, the respondent referred to the written protocol when answering survey questions. The respondent also qualified any aspect of the protocol that was unspecific or unclear. Fifty-two $(91.2 \%)$ units with guidelines recommended sweetening agents for venipuncture/venous cannulation, followed by 50 $(87.7 \%)$ for lumbar puncture and $47(82.5 \%)$ for heel lancing, respectively (Table 1). In $24(42.1 \%)$ units, a pacifier was always given with the sweetener.

The dose for sucrose ranged from $0.05 \mathrm{~mL}$ of $24 \%$ solution to $3 \mathrm{~mL}$ of $25 \%$ solution. In $80 \%$ of the units, the sucrose dosage regimen varied according to infant gestational age or weight. For infants $<31$ weeks' gestation or $<1500 \mathrm{~g}$, the most commonly recommended dose was $0.5 \mathrm{~mL}$ of $24 \%$ solution, and for $\geq 31$ weeks' gestation or
TABLE 1. Recommended Use of Sweetening Agents for Different Procedures $(\mathrm{N}=57)$

\begin{tabular}{lr}
\hline Procedures & No. (\%) \\
\hline Venipuncture & $52(91.2)$ \\
Venous cannulation & $52(91.2)$ \\
Lumbar puncture & $50(87.7)$ \\
Heel lance & $47(82.5)$ \\
Intramuscular injection & $45(78.9)$ \\
Arterial puncture & $43(75.4)$ \\
Subcutaneous injection & $42(73.7)$ \\
Dressing change & $38(66.7)$ \\
Urinary catheterization & $36(63.2)$ \\
Suprapubic aspiration & $34(59.6)$ \\
Eye examination & $33(57.9)$ \\
Chest tube & $32(56.1)$ \\
Percutaneous central venous catheter placement & $32(56.1)$ \\
Nasogastric tube insertion & $13(22.8)$ \\
Tracheal suctioning & $8(14.0)$ \\
Endotracheal tube insertion & $3(5.3)$ \\
\hline
\end{tabular}

$\geq 1500 \mathrm{~g}$, it was $1 \mathrm{~mL}$ of $24 \%$ solution. The guidelines restricted the use of sweetening agents to infants of $\geq 27$ to 28 weeks' gestation in 11 units, and $\geq 30$ to 32 weeks' gestation in 13 units.

A limit on the maximum number of doses that could be administered during a single procedure was present in $23(40.4 \%)$ units, and ranged from 1 to 4 (mode, 1 ). In addition, $42(73.7 \%)$ units reported a maximum daily number of doses that ranged from 2 to 24 (mode, 6). The most common contraindications for the use of sweetening agents included proven/suspected necrotizing enterocolitis and nil per os, in $44(77.2 \%)$ and $42(75 \%)$ units, respectively (Table 2). Logistic regression analysis did not identify any factors that significantly predicted the reported utilization of sweetening agents.

\section{DISCUSSION}

The present study demonstrated that two-thirds of special care nurseries and neonatal intensive care units across Canada had clinical practice guidelines that support the use of sweetening agents for procedural pain management. This rate is almost 3 times higher than a 2004 survey in Australia, ${ }^{11}$ but falls short of currently published consensus guidelines and position statements that recommend use of sweetening agents in all infants undergoing procedures. ${ }^{9}$

TABLE 2. Contraindications for Use of Sweetening Agents $(\mathrm{N}=57)$

\begin{tabular}{ll}
\hline Characteristic & No. $(\mathbf{\%})$ \\
\hline Necrotizing enterocolitis & $44(77.2)$ \\
Nil per os (NPO)* & $42(75.0)$ \\
Glucose intolerance & $21(36.8)$ \\
Concurrent opioid use & $21(36.8)$ \\
Specific gestational age group & $18(31.6)$ \\
Infant of diabetic mother* & $16(28.6)$ \\
Parental refusal & $12(21.1)$ \\
\hline
\end{tabular}

$* \mathrm{~N}=56$ 
In addition, the present study determined that the dosing regimens for sweeteners were highly variable, and restrictions were often placed on the characteristics of infants that were eligible to receive them. Thus, the presence of a practice guideline did not ensure that all infants were eligible to receive sweetening agents before all painful medical procedures.

We hypothesize that the variability in clinical practice guidelines is due to gaps in knowledge about the pharmacology of sweetening agents, including the mechanism of action, optimal dose, and safety of repeated does. ${ }^{9}$ That there is no consensus about what constitutes the "appropriate use of sweetening agents" among units has implications for the interpretation of pain audits that document actual utilization patterns of analgesic agents. Pain management audits are usually discussed in terms of barriers in knowledge, attitudes, and beliefs. ${ }^{14}$ It is clear from the results of this study that utilization patterns for sweetening agents need to be interpreted within the context of acceptable practices in individual units rather than externally published consensus statements.

The strengths of the present study are the very high response rate $(93.5 \%)$ and validation of protocols for analgesic use. In contrast, the previous survey achieved a relatively low response rate $(58 \%)$, and did not validate responses. ${ }^{11} \mathrm{We}$ did not measure actual utilization of sweetening agents, and therefore cannot comment on the proportion of infants given sweetening agents relative to guideline recommendations. This is clearly an important area for future study.

In conclusion, this study demonstrated that two-thirds of Canadian special care units and neonatal intensive care units have practice guidelines in place for the use of sweetening agents to manage procedural pain in neonates. There are wide variations in prescribing guidelines, resulting in the potential for many infants to undergo procedures without sweetening agent analgesia. Further study of the pharmacology of sweeteners is needed to answer the current gaps in research knowledge that will in turn, be used to modify and improve practice guidelines.

\section{ACKNOWLEDGMENT}

The authors are thankful to the respondents for their participation.

\section{REFERENCES}

1. Simons SHP, van Dijk M, Anand KS, et al. Do we still hurt newborn babies? A prospective study of procedural pain and analgesia in neonates. Arch Pediatr Adolesc Med. 2003;157: $1058-1064$.

2. Taddio A, Katz J. The effects of early pain experience in full-term and preterm neonates on subsequent pain responses later in infancy and childhood. Pediatr Drugs. 2005;7: 245-257.

3. Grunau RE. Long-term consequences of pain in human neonates. In: Anand KJS, Stevens BJ, McGrath PJ, eds. Pain in Neonates. 2nd revised and enlarged edition. Amsterdam: Elsevier; 2000:55-76.

4. Anand KJ, Johnston CC, Oberlander TF, et al. Analgesia and local anesthesia during invasive procedures in the neonate. Clin Ther. 2005;27:844-876.

5. Stevens B, Yamada J, Ohlsson A. Sucrose for analgesia in newborn infants undergoing painful procedures. Cochrane Database Syst Rev. 2004;CD001069.

6. Blass EM, Watt LB. Suckling and sucrose-induced analgesia in human newborns. Pain. 1999;83:611-623.

7. American Academy of Pediatrics. Committee on Fetus and Newborn. Committee on Drugs. Section on Anesthesiology. Section on Surgery. Canadian Paediatric Society. Fetus and Newborn Committee. Prevention and management of pain and stress in the neonate. Pediatrics. 2000;105:454-461.

8. Anand KJ. Consensus statement for the prevention and management of pain in the newborn. Arch Pediatr Adolesc Med. 2001;155:173-180.

9. American Academy of Pediatrics and Canadian Paediatric Society. Prevention and management of pain in the neonate: an update. Pediatrics. 2006;118:2231-2241

10. Lefrak L, Burch K, Caravantes R, et al. Sucrose analgesia: identifying potentially better practices. Pediatrics. 2006;118: S197-S202

11. Harrison D, Loughnan P, Johnston L. Pain assessment and procedural pain management practices in neonatal units in Australia. J Paediatr Child Health. 2006;42:6-9.

12. Woodward CA, Chambers LW. Guide to Questionnaire Construction and Question Writing. Ottawa: Canadian Public Health Association; 1986.

13. Aday LA, Cornelius LJ. Designing and Conducting Health Surveys: a Comprehensive Guide. 3rd ed. San Francisco: JosseyBass; 2006.

14. Taylor EM, Boyer K, Campbell FA. Pain in hospitalized children: a prospective cross-sectional survey of pain prevalence, intensity, assessment and management in a Canadian pediatric teaching hospital. Pain Res Manage. 2008; $13: 25-32$. 